\title{
Heat shock proteins 70 and 90 from Clonorchis sinensis induce Th1 response and stimulate antibody production
}

Eun Joo Chung, Young-II Jeong, Myoung-Ro Lee, Yu Jung Kim, Sang-Eun Lee, Shin-Hyeong Cho, Won-Ja Lee, Mi-Yeoun Park and Jung-Won Ju*

\begin{abstract}
Background: Heat shock proteins (HSPs) are found in all prokaryotes and most compartments of eukaryotic cells. Members of the HSP family mediate immune responses to tissue damage or cellular stress. However, little is known about the immune response induced by the oriental liver fluke, Clonorchis sinensis, even though this organism is carcinogenic to humans. We address this issue in the present study in mouse bone marrow dendritic cells (mBMDCs), using recombinant HSP70 and 90 from C. sinensis (rCsHSP70 and rCsHSP90).

Methods: $\mathrm{rCsHSP70}$ and $\mathrm{rCSHSP90}$ were produced in an E. coli system. Purified recombinant proteins were treated in BMDCs isolated from C57BL/6 mice. T cells were isolated from Balb/c mice and co-cultured with activated mBMDCs. Expression of surface molecules was measured by flow cytometry and cytokine secretion was quantified using ELISA. C57BL/6 mice were divided into four groups, including peptide alone, peptide/Freund's adjuvant, peptide/CsHSP70, peptide/CsHSP90, and were immunized intraperitoneally three times. Two weeks after final immunization, antibodies against peptide were measured using ELISA.

Results: Both proteins induced a dose-dependent upregulation in major histocompatibility complex and co-stimulatory molecule expression and increased secretion of pro-inflammatory cytokines including interleukin (IL)-1 $\beta,-6$, and -12p70 and tumor necrosis factor-a in mBMDCs. Furthermore, when allogenic T cells were incubated with mBMDCs activated by rCsHSP70 and rCsHSP90, the helper T cell (Th)1 cytokine interferon- $\gamma$ was up-regulated whereas the level of the Th2 cytokine IL-4 was unchanged. These results indicate that rCSHSPs predominantly induce a Th1 response. Over and above these results, we also demonstrated that the production of peptide-specific antibodies can be activated after immunization via in vitro peptide binding with rCsHSP70 or rCsHSP90.
\end{abstract}

Conclusion: This study showed for the first time that the HSP or HSP/peptide complexes of $C$. sinensis could be considered as a more effective vaccine against $C$. sinensis infection as results of the activator of host immune response as well as the adjuvant for antigenic peptide conjugate to induce peptide-specific antibody response in mice.

Keywords: Clonorchis sinensis, Heat shock protein, Immunogenicity, Peptide, Adjuvant

\footnotetext{
*Correspondence: jupapa@korea.kr

Division of Malaria and Parasitic Diseases, Center for Immunology and

Pathology, National Research Institute of Health, Korea Centers for Disease

Control \& Prevention, Osong 28159, Republic of Korea
} 


\section{Background}

Clonorchis sinensis is a fish-borne parasitic trematode widely distributed in Korea, China, Taiwan, Vietnam, and Russia that is the causative agent of human clonorchiasis [1], which mainly occurs as a result of eating raw or undercooked freshwater fish infested with the metacercariae of $C$. sinensis [2-4]. Eating uncooked fish is also an important risk factor for intrahepatic CCA caused by clonorchiasis [5]. In 2009, C. sinensis was classified as a Group 1 carcinogen in humans by the International Agency for Research on Cancer [6]. The most common cause of death by clonorchiasis is cholangiocarcinoma (CCA) [7], a cancer of the bile ducts that is associated with chronic and severe liver fluke infection [1]; continuous stimulation of the bile duct by this organism induces pathological changes such as biliary mucosal hyperplasia, bile duct enlargement, periductal fibrosis, mechanical obstruction, inflammation, epithelial adenomatous hyperplasia, and biliary cirrhosis $[1,2,8]$. The association between liver flukes and CCA has also been demonstrated animal models [9]. Advanced CCA typically has poor prognosis, with a median survival time of $<24$ months $[5,10]$; around 5,000 cases of CCA attributed to C. sinensis infection are reported worldwide each year [11]. A survey in Korea showed that the ratio of patients with CCA differed significantly between those with and without clonorchiasis [12]. As such, considerable effort has been focused on the development of a vaccine against $C$. sinensis. One study reported the protective effect of Cs14-3-3 epsilon protein [13] while another showed that CsTP22.3 expressed by Bacillus subtilis spores was immunogenic and could be orally administered to provide protection against $C$. sinensis infection [14].

Heat shock proteins (HSPs) constitute a highly conserved family in most organisms that provide cellular protection under stressful conditions including heat, oxidative stress, glucose starvation, irradiation and viral infection [15]. HSP70 and HSP90 provide a link between innate and adaptive immune responses via activation of lymphocytes and antigen-presenting cells (APCs) such as dendritic cells (DCs) $[15,16]$. HSPs purified from bacterial and mammalian sources are potent stimulators of the innate immune response [16], which includes production of pro-inflammatory cytokines such as tumor necrosis factor (TNF)- $\alpha$ and interleukin (IL)-1 $\beta,-6$, and -12 by macrophages [17] and DCs [18, 19] and the upregulation of surface markers such as cluster of differentiation (CD)40, CD80, and CD86 in DCs [20]. HSPs also induce antigen-dependent $\mathrm{T}$ cell activation as well as interferon (IFN) $-\gamma$ secretion $[21,22]$, and are associated with peptides that are presented by major histocompatibility complex (MHC), MHC II and MHC I on APCs [23-25]. Antigenic peptide/HSP70 complexes activate DCs for cytokine release and prime cytotoxic T lymphocyte (CTL) responses [18, 26]. Mice immunized with HSP/CTL epitope peptide complexes produced CTLs and showed a peptide-specific antibody response [27]. Various studies have shown that immunization with parasite HSPs induce cellular and humoral immune responses and protect against infection by the parasite [20, 28-31]. These findings suggest that pathogen HSPs are a candidate vaccine against infectious diseases. However, no studies have been done on host immune responses to HSPs in trematodes, including C. sinensis despite its risk to humans.

We addressed this in the present study by investigating in vitro immune responses to C. sinensis HSP70 and HSP90. Their adjuvant effect was confirmed by analyzing antipeptide antibody production after immunization of peptide bound to CsHSP70 and CsHSP90 in a murine model.

\section{Methods}

Recombinant $\mathrm{C}$. sinensis ( $\mathrm{rCsHSP}$ ) expression and purification cDNA clones encoding CsHSP70 and CsHSP90 were obtained from the adult $C$. sinensis cDNA library. The open reading frames of the two proteins were PCRamplified using the following forward and reverse primer sets: CsHSP70, 5'-GAG CGA TCT CAT GTC GAA GGT CAT GCT-3' and 5'-CGC GTC GAC CTA TTG TTT CTG CTG AG-3'; and CsHSP90, 5'-GCA ATT CCA TAT GTC TTG CGA ACC GAT GGC-3' and 5'-C CC AAG CTT ATC GAC TTC TTC CAT TCC AGC-3'. The PCR products were cloned into the $\mathrm{pET} 28 \mathrm{a}$ and pET21a vectors (Novagen, Madison, WI, USA), respectively. Recombinant plasmids were transformed into Escherichia coli BL21 (DE3)-RIPL cells, which were grown in Luria-Bertani medium containing ampicillin or kanamycin. Isopropyl-B-D-thiogalactopyranoside was added to the culture to a final concentration of $0.5 \mathrm{mM}$ and cells were incubated for $20 \mathrm{~h}$ at $16{ }^{\circ} \mathrm{C}$, then harvested by centrifugation and resuspended in lysis buffer $\left[50 \mathrm{mM} \mathrm{NaH}{ }_{2} \mathrm{PO}_{4}\right.$, $300 \mathrm{mM} \mathrm{NaCl}$, and $5 \mathrm{mM}$ imidazole $(\mathrm{pH}$ 8.0)]. The cell suspension was sonicated on ice, and supernatants were collected after centrifugation. Recombinant proteins were purified using Ni-nitrilotriacetic acid agarose (Qiagen, Valencia, CA, USA) under native conditions. Briefly, supernatants were loaded in a column pre-equilibrated with lysis buffer. The column was washed with five bed volumes of wash buffer [50 mM NaH $\mathrm{mO}_{4}, 300 \mathrm{mM} \mathrm{NaCl}$, and $20 \mathrm{mM}$ imidazole $(\mathrm{pH}$ 8.0)] and then eluted with elution buffer [50 mM NaH${ }_{2} \mathrm{PO}_{4}, 300 \mathrm{mM} \mathrm{NaCl}, 250 \mathrm{mM}$ imidazole ( $\mathrm{pH}$ 8.0)]. The eluted protein fraction was concentrated using Amicon Ultra-4 centrifugal filter devices (Millipore, Billerica, MA, USA) and protein concentration was estimated by the Bradford method using bovine serum albumin as a standard. The purity of eluted proteins was evaluated by sodium dodecyl sulfate polyacrylamide gel electrophoresis (SDS-PAGE) and sequences were confirmed by liquid chromatography-tandem mass spectrometry (LC-MS/MS). Recombinant proteins were dialyzed against Dulbecco's phosphate buffered saline (DPBS; 
$\mathrm{pH}$ 7.4) for mouse immunization or treatment of mouse bone marrow dendritic cells (mBMDCs).

Before experiments, endotoxin contaminants of rCsHSP70 and rCsHSP90 were removed using Detoxi-Gel endotoxin removal columns (Pierce, Rockford, IL, USA). The endotoxin level in recombinant proteins was determined with the Limulus amoebocyte lysate (LAL) Chromogenic Endotoxin Quantitation kit (Pierce) and was found to be negligible $(<1 \mathrm{EU} / \mathrm{ml})$. Additionally, $\mathrm{rCsHSPs}$ were pre-incubated with polymyxin B (Sigma-Aldrich, St. Louis, MO, USA) before being used for mBMDC treatment.

\section{mBMDC preparation}

Femurs and tibiae were removed from C57BL/6 mice and washed with DPBS. Both ends of isolated bones were cut with scissors and the bone marrow was flushed out with ice-cold DPBS using a syringe. After one wash with DPBS, erythrocytes were lysed by treatment with red blood cell lysis buffer (Sigma-Aldrich). mBMDCs were obtained by culturing the cells $\left(2 \times 10^{6}\right.$ cells $\left./ \mathrm{ml}\right)$ in the presence of $20 \mathrm{ng} / \mathrm{ml}$ recombinant granulocyte-macrophage colonystimulating factor (GM-CSF; Peprotech, Rocky Hill, NJ, USA) for 8 days in RF10 medium (Roswell Park Memorial Institute (RPMI)-1640 medium (Gibco, Carlsbad, CA, USA) supplemented with $10 \%$ fetal bovine serum (FBS; Gibco), $1 \%$ penicillin-streptomycin, and L-glutamine). The medium was replaced every 3 days.

Analysis of DC-secreted cytokines and DC surface markers After 8 days, cells were harvested and resuspended in RF10 medium containing GM-CSF. Cells were then seeded in a 6-well plate at a density of $2 \times 10^{6}$ cells/well. rCsHSPs or proline-rich (ProR) peptide were mixed with $50 \mu \mathrm{g} / \mathrm{ml}$ polymyxin $\mathrm{B}$ and incubated at $4{ }^{\circ} \mathrm{C}$. After $1 \mathrm{~h}$, immature DCs were stimulated with $\mathrm{rCsHSP70,} \mathrm{rCsHSP90,} \mathrm{ProR} \mathrm{peptide,}$ or $0.1 \mu \mathrm{g} / \mathrm{ml}$ lipopolysaccharide (LPS; Sigma-Aldrich) as a positive control for $18 \mathrm{~h}$ in humidified $5 \% \mathrm{CO}_{2}$ incubator at $37^{\circ} \mathrm{C}$. After treatment, cells were harvested and centrifuged at $2000 \times g$ for $5 \mathrm{~min}$. The supernatant was collected and stored at $-20{ }^{\circ} \mathrm{C}$ until use. Cells were then labeled with fluorescein isothiocyanate (FITC)-conjugated anti-mouse CD11c antibody; phycoerythrin (PE)-conjugated antimouse CD40, CD80, or CD86 antibody; or allophycocyanin (APC)-conjugated MHC Class II (I-A/I-E) or MHC Class I (H-2 kb) antibody (all from eBioscience, San Diego, CA, USA) for $30 \mathrm{~min}$ at $4{ }^{\circ} \mathrm{C}$. After $30 \mathrm{~min}$ of labeling, cells were washed with DPBS and resuspended in $4 \%$ paraformaldehyde for analysis with a FACSVerse flow cytometer (BD Biosciences, Franklin Lakes, NJ, USA); data were analyzed using FlowJo software (Ashland, OR, USA). Levels of interleukin (IL)-6, $-1 \beta,-12$ p70, and -10 and TNF- $\alpha$ in culture supernatants were quantified with an enzyme-linked immunosorbent assay (ELISA) kit (R\&D Systems,
Minneapolis, MN, USA), according to the manufacturer's instructions.

\section{Measurement of intracellular cytokine levels in DCs} Intracellular cytokines were detected using the Cytofix/ Cytoperm kit (BD Biosciences), according to the manufacturer's instructions. Briefly, DCs $\left(1 \times 10^{6}\right.$ cells $\left./ \mathrm{ml}\right)$ treated with rCsHSPs were harvested and centrifuged at $1500 \times \mathrm{rpm}$ for $5 \mathrm{~min}$. The supernatant was removed and the pellet was washed with staining buffer (2.5\% FBS in DPBS), and $1 \mu \mathrm{g} / \mathrm{ml}$ of FITC-conjugated anti-mouse CD11c IgG (eBioscience) was added followed by incubation at $4{ }^{\circ} \mathrm{C}$ for $30 \mathrm{~min}$. The pellets were washed twice with staining buffer and cells were collected by centrifugation. The pellets were resuspended and incubated in $250 \mu \mathrm{l}$ of Cytofix/Cytoperm solution (BD Biosciences) for $20 \mathrm{~min}$ at $4{ }^{\circ} \mathrm{C}$, then washed twice in $1 \times$ Perm/Wash solution (BD Biosciences). To label intracellular cytokines, cells were incubated in $1 \mu \mathrm{g} / \mathrm{ml} \mathrm{PE-conjugated}$ anti-mouse IL-10 or-12 or IFN- $\gamma$ IgG solution (eBiosciences) at $4{ }^{\circ} \mathrm{C}$ for $30 \mathrm{~min}$ in the dark, then washed twice in $1 \times$ Perm/Wash solution and fixed with $4 \%$ paraformaldehyde at $4{ }^{\circ} \mathrm{C}$. The fluorescence intensity of intracellular cytokines was measured by flow cytometry.

\section{Endocytosis test}

To analyze the endocytic capacity of DCs, $1 \times 10^{6}$ cells were incubated in the presence or absence of rCsHSPs for $20 \mathrm{~h}$. The cells were harvested and washed twice with DPBS, and $1 \times 10^{6}$ cells were incubated at $37^{\circ} \mathrm{C}$ or $4{ }^{\circ} \mathrm{C}$ for an additional $30 \mathrm{~min}$ with $1 \mathrm{mg} / \mathrm{ml}$ FITC-dextran (40,000 MW anionic, lysine-fixable; Molecular Probes, Eugene, OR, USA). After incubation, cells were washed twice with ice-cold staining buffer $\left(0.1 \%\right.$ FBS and $0.01 \% \mathrm{NaN}_{3}$ in PBS), and $1 \mu \mathrm{g} / \mathrm{ml}$ PE-conjugated anti-mouse CD11c IgG (eBioscience) was added followed by incubation at $4{ }^{\circ} \mathrm{C}$ for $30 \mathrm{~min}$. After two washes, labeled DCs were analyzed by flow cytometry.

\section{Allogenic mixed lymphocyte reaction (MLR)}

$\mathrm{T}$ cells were prepared from splenocytes obtained by grinding spleens of 8 -week-old BALB/c mice. $\mathrm{CD}^{+} \mathrm{T}$ cells were purified via negative selection using magneticactivated cell sorting (MACS) columns (Pan T Cell Isolation Kit II; Miltenyi Biotec, San Diego, CA, USA). Isolated $\mathrm{T}$ cells were stained with carboxyfluorescein succinimidyl ester (CFSE) using the Cell Trace CFSE Cell Proliferation kit (Molecular Probes). BMDCs $(5 \times$ $10^{4}$ cells/well) were stimulated with $10 \mu \mathrm{g} / \mathrm{ml} \mathrm{rCsHSPs}$ for $24 \mathrm{~h}$, washed twice, and then co-cultured with CFSElabeled T cells $\left(5 \times 10^{5}\right.$ cells/well $)$. Mixed cells were added to a 96-well round-bottom plate and cultured in humidified $5 \% \mathrm{CO}_{2}$ incubator at $37^{\circ} \mathrm{C}$. Cells were harvested after 4, 5, and 6 days and supernatants were collected and stored at $-20{ }^{\circ} \mathrm{C}$ until analysis. Harvested cells were washed twice 
and then stained with $1 \mu \mathrm{g} / \mathrm{ml} \mathrm{PE}$-conjugated anti-mouse CD3e antibody (eBioscience) at $4{ }^{\circ} \mathrm{C}$ for $30 \mathrm{~min}$. After two washes with DPBS, the cells were resuspended in $4 \%$ paraformaldehyde and fluorescence intensity was measured by flow cytometry. IL- 2 and -4 and IFN- $\gamma$ concentrations in the cell culture supernatant were measured with ELISA kits (R\&D Systems), according to the manufacturer's instructions.

\section{rCsHSPs immunization and measurement of cytokines secreted from splenocytes}

Groups of 5 C57BL/6 mice were immunized intraperitoneally with $50 \mu \mathrm{g}$ of $\mathrm{rCsHSP70}$ or rCsHSP90 emulsified in complete Freund's adjuvant (CFA). Subsequent booster doses were given on 14th and 28th day with $50 \mu \mathrm{g}$ of rCsHSP70 or rCsHSP90 emulsified in incomplete Freund's adjuvant (IFA). The control mice received an equal volume of PBS mixed with adjuvant. After the 7th day of the last immunization, the splenocytes were isolated from mice and cultured $(1 \times$ $10^{5}$ cells/well) either in the absence or presence of $5 \mu \mathrm{g} / \mathrm{ml}$ of rCsHSPs for $72 \mathrm{~h}$. The levels of IL-2, -4 and IFN- $\gamma$ in culture supernatants were determined using ELISA kits (R\&D system), according to the manufacturer's instructions.

\section{rCsHSPs/CsProR peptide complex formation}

CsProR peptide (> 95\% purity; DAPVPKSGGPDAPVP KSGGPDAPVPKSGG) was synthesized by Peptron (Daejeon, Korea). The ProR sequence was derived from the proline-rich antigen of C. sinensis (GenBank accession no. AAK35165). To prepare rCsHSPs/CsProR peptide complexes, CsHSP70 or CsHSP90 was mixed with CsProR peptide for $1 \mathrm{~h}$ in binding buffer [ $1 \mathrm{mM}$ ADP and $1 \mathrm{mM} \mathrm{MgCl}_{2}$ in DPBS (pH 7.4)] at $37{ }^{\circ} \mathrm{C}$ with shaking. After $1 \mathrm{~h}$ of incubation, $50 \mu \mathrm{g} / \mathrm{ml}$ polymyxin $\mathrm{B}$ was added, and complexes were incubated at $4{ }^{\circ} \mathrm{C}$ for $1 \mathrm{~h}$, then immediately transferred to ice until use.

\section{Immunization with CsHSPs/ProR peptide complexes}

C57BL/6 mice were divided into four groups of five animals each as follows: group 1 was immunized with ProR peptide alone; group 2 was immunized with ProR peptide combined with Freund's adjuvant (FA); group three was immunized with CsHSP70/ProR peptide complexes; and group four was immunized with CsHSP90/ProR peptide complexes. Mice were intraperitoneally injected three times at 2-week intervals with complete FA for the first injection and incomplete FA for the first and second boosts. After final immunization, whole blood was collected from each mouse by cardiac puncture and centrifuged at $2000 \times \mathrm{rpm}$ and $4{ }^{\circ} \mathrm{C}$ for $10 \mathrm{~min}$. Obtained serum samples were stored at $-20^{\circ} \mathrm{C}$ until analysis.

\section{Analysis of T cell proliferation}

Splenocytes were prepared by grinding the spleens of five immunized mice from each group. $\mathrm{T}$ cells were purified from splenocytes via negative selection using MACS columns. Prepared T cells were cultured in a 96-well plate at a density of $1 \times 10^{5}$ cells/well in a final volume of $100 \mu \mathrm{l} /$ well after resuspension in cell culture medium (RPMI 1640 with 10\% FBS and 1\% antibiotics). The plate was incubated in the absence or presence of ProR peptide $(10 \mu \mathrm{g} / \mathrm{ml})$ at $37{ }^{\circ} \mathrm{C}$ in a $5 \% \mathrm{CO}_{2}$ incubator for $72 \mathrm{~h}$ before $10 \mu \mathrm{l} /$ well WST-1/electro coupling solution (BioVision, Milpitas, CA, USA) was added to each well. The cells were incubated under standard culture conditions for $4 \mathrm{~h}$, and sample absorbance was measured using a microplate reader at $450 \mathrm{~nm}$ (reference wavelength: $620 \mathrm{~nm}$ ).

\section{Determination of antibody titer}

Humoral immune responses elicited by ProR peptide/ rCsHSPs complexes were analyzed by detecting the production of specific antibodies against the peptide in CsProR peptide-coated ELISA plates. Briefly, streptavidincoated 96-well plates (Thermo Fisher Scientific, Waltham, MA, USA) were incubated with $5 \mu \mathrm{g} / \mathrm{ml}$ biotin-conjugated CsProR peptide for $18 \mathrm{~h}$ at $4{ }^{\circ} \mathrm{C}$. All subsequent steps were carried out at room temperature. After three washes with PBS containing $0.1 \%$ Triton X-100 (PBST), plates were blocked with blocking buffer (5\% skim milk in PBST) for $2 \mathrm{~h}$, washed, and incubated in immunized mouse serum diluted in blocking buffer for $2 \mathrm{~h}$. After washing, $100 \mu \mathrm{l} /$ well horseradish peroxidase-conjugated rabbit anti-mouse IgG (Sigma-Aldrich) diluted 1:10,000 in blocking buffer were added, followed by incubation for $2 \mathrm{~h} ; 50 \mu \mathrm{l} /$ well 3,3',5,5'tetramethylbenzidine $/ \mathrm{H}_{2} \mathrm{O}_{2}$ substrate were added, followed by incubation for $30 \mathrm{~min}$. The enzymatic reaction was terminated by adding $50 \mu \mathrm{l} /$ well of $2 \mathrm{~N} \mathrm{H}_{2} \mathrm{SO}_{4}$ to the wells and optical density was measured with a spectrophotometer at $450 \mathrm{~nm}$ (reference wavelength: $620 \mathrm{~nm}$ ). For measuring IgG isotypes, detection of antibodies was determined using either horseradish peroxidase-conjugated goat anti-mouse IgG1 or IgG2a (BioRad, Hercules, CA, USA).

\section{Statistics analysis}

Statistical analyses were performed using two-tailed $t$-tests or 2 way ANOVA run on GraphPad Prism version 5.01 software (GraphPad Software, San Diego, CA). Experimental data were presented as the standard error of the mean (SEM). Differences between groups were considered significant if $P$-value was less than 0.05 .

\section{Results}

\section{Purification of rCsHSP70 and rCsHSP90}

CsHSP70 and CsHSP90 are 656 and 714 amino acids, respectively, with predicted molecular weights of 75 and $83.3 \mathrm{kDa}$, respectively. The expression of these proteins was confirmed by the band size in the SDS-PAGE analysis (Fig. 1); the purified proteins had significant 

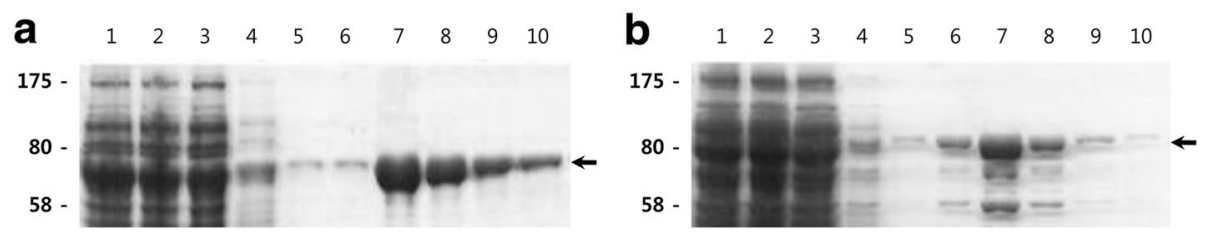

Fig. 1 SDS-PAGE images of rCSHSPs expression. a rCSHSP70 and b rCSHSP90 were purified under native conditions and resolved by 10\% SDS-PAGE. Molecular weight markers are indicated in left. Lane 1: total induced cell lysate; Lane 2: supernatant (sup); Lane 3: flow through; Lanes 4-5: wash; Lanes 6-10: eluted fraction. rCsHSPs are indicated by arrows

homology $(P<0.05)$ to the known sequences of CsHSP70 and CsHSP90, as determined by LC-MS/MS.

\section{rCsHSPs increase surface marker expression and cytokine production in $\mathrm{mBMDCs}$}

rCsHSP70 and rCsHSP90 with endotoxins removed induced the expression of co-stimulatory molecules by mBMDCs in a dose-dependent manner (Fig. 2). rCsHSP induced mBMDC maturation, as demonstrated by the upregulation of the co-stimulatory molecules CD40,
CD80, and CD86, whereas treatment with either protein dose-dependently increased MHC I and MHC II expression. Moreover, MHC I expression was higher than in the LPS-treated positive control group.

Cytokines secreted from activated DCs are classified as Th2-type cytokines (e.g., IL-10) or pro-inflammatory cytokines (e.g., IL-12p70, -6 , and $-1 \beta$ and TNF- $\alpha$ ) $[18,32]$. We measured the concentration of these cytokines secreted from mBMDCs by ELISA in cell culture supernatants. There was a dose-dependent increase in the

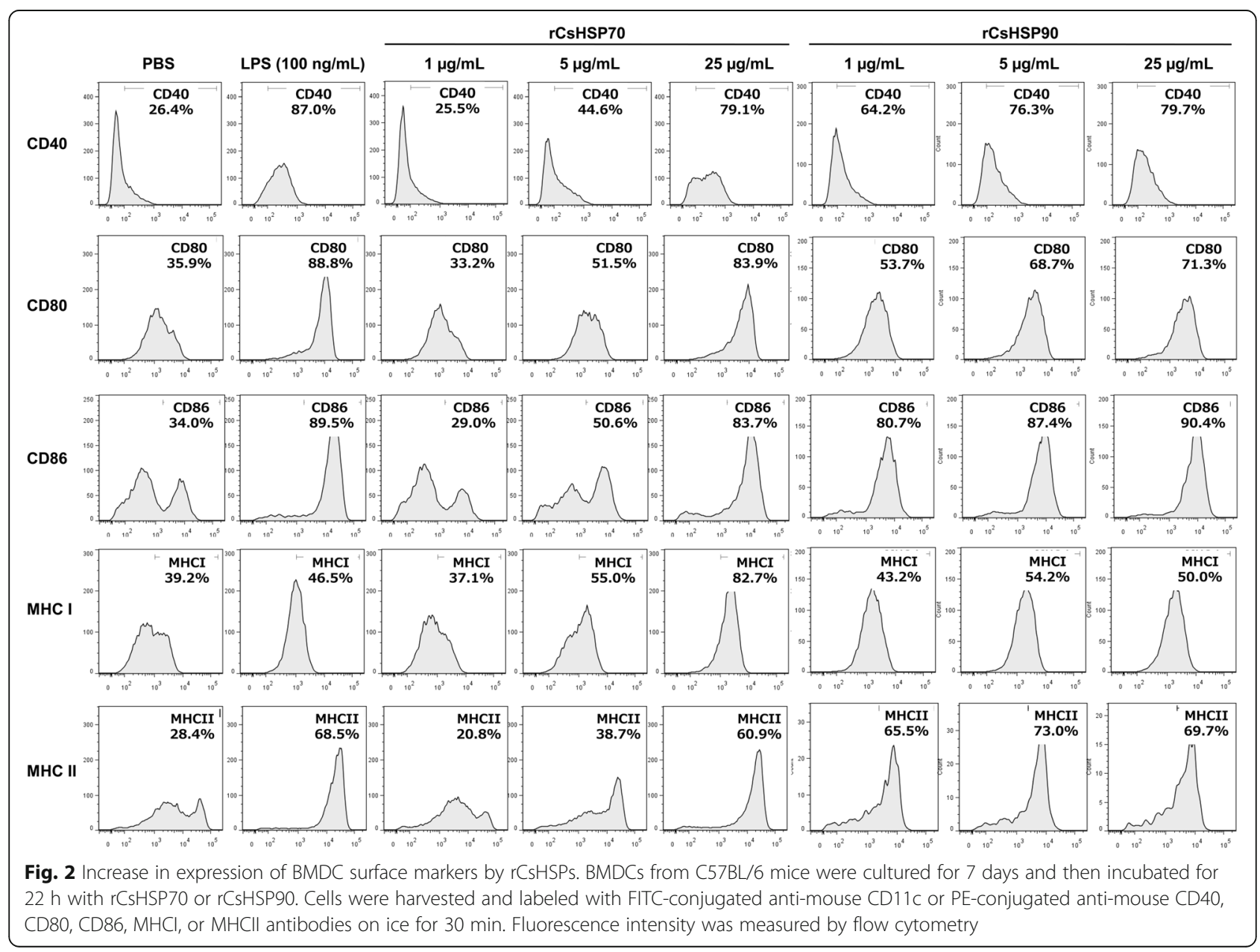




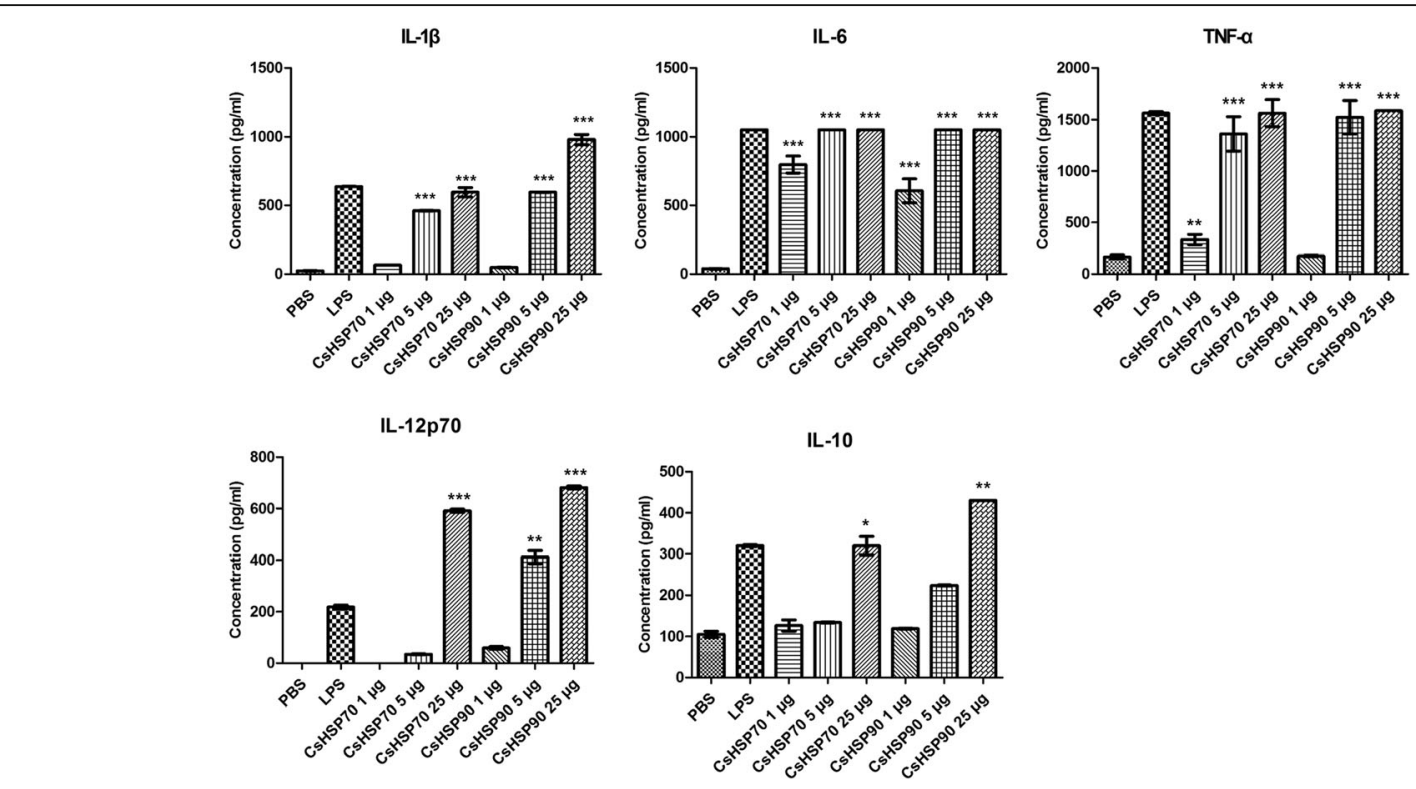

Fig. 3 rCsHSPs dose-dependently induce cytokine secretion by BMDCs. BMDCs from C57BL/6 mice were cultured for 7 days and then incubated for $22 \mathrm{~h}$ with rCSHSP70 or rCsHSP90. Secreted cytokine levels in culture supernatants were measured by ELISA. The results are shown as the mean \pm standard error (SEM). ${ }^{*} P<0.05,{ }^{* *} P<0.01,{ }^{* *} P<0.001$ compared to PBS-treated group

secreted levels of pro-inflammatory cytokines (IL-12p70, -6 , and $-1 \beta$ and TNF- $\alpha$ ) following treatment with rCsHSP70 $\left(t_{(2)}=12.25, P=0.0066\right)$ or $\mathrm{rCsHSP90}\left(t_{(2)}=\right.$ $16.22, P=0.0038)$. IL-10 level was also slightly increased by high concentrations of rCsHSP70 $\left(t_{(2)}=\right.$ 9.065, $P=0.012)$ or $\mathrm{rCsHSP90}\left(t_{(2)}=27.01, P=0.0014\right)$ (Fig. 3). In addition, measurement of intracellular IL-10 and -12 production by flow cytometry revealed an increase in IL-12 level according to rCsHSP concentration, whereas IL-10 was not detected (Fig. 4).

\section{rCsHSP70 and rCsHSP90 are taken up as antigens by mBMDCs}

DCs mature following antigen uptake and subsequently decrease their endocytic activity [33, 34]. We investigated whether rCsHSPs affect the endocytic capacity of

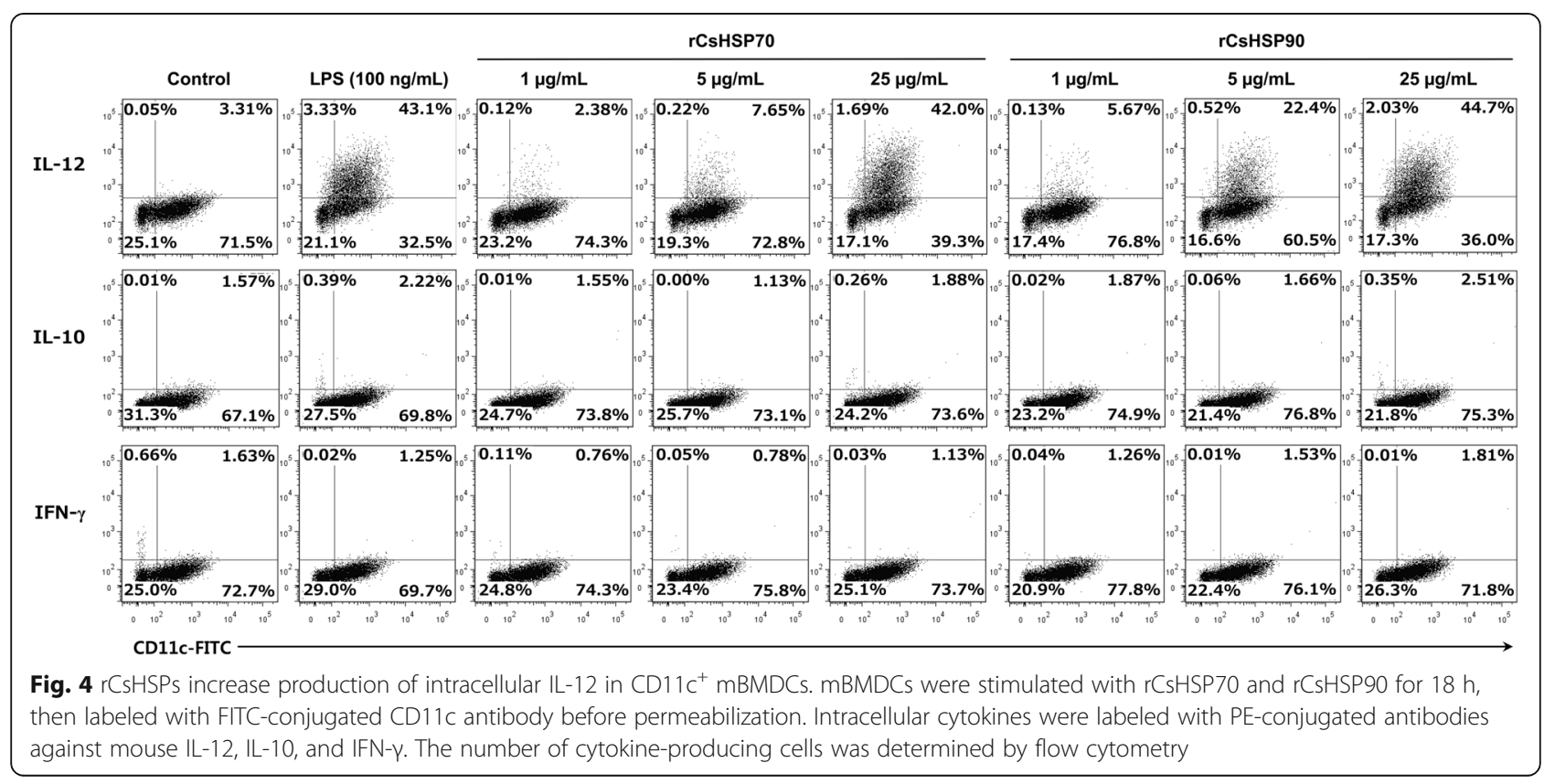


mBMDCs treated with FITC-conjugated dextran and activated with rCsHSPs (Fig. 5). Immature mBMDCs captured a large amount of dextran-FITC at $37{ }^{\circ} \mathrm{C}$ relative to the non-specific control at $4{ }^{\circ} \mathrm{C}$. This uptake was abrogated upon treatment with $5 \mu \mathrm{g} / \mathrm{ml} \mathrm{rCsHSPs}$ to a level similar to that induced by the positive control LPS $(0.1 \mu \mathrm{g} / \mathrm{ml})$.

\section{mBMDCs activated by CsHSP70 and CsHSP90 induce T cell proliferation and differentiation}

To further investigate the cellular response elicited by rCsHSPs, we evaluated $\mathrm{T}$ lymphocyte proliferation and cytokine secretion in response to stimulation with $5 \mu \mathrm{g} /$ $\mathrm{ml} \mathrm{rCsHSPs.} \mathrm{mBMDCs} \mathrm{activated} \mathrm{with} \mathrm{rCsHSPs}$ were cocultured with $\mathrm{T}$ cells isolated from splenocytes of allogenic mice; $\mathrm{T}$ cell activation was confirmed by MLR. The number of $\mathrm{T}$ cells stimulated with mature BMDCs increased in a time-dependent manner (Fig. 6a). In addition, IL-2 (CsHSP70; $t_{(2)}=5.322, P=0.0335$, CsHSP90; $\left.t_{(2)}=5.257, P=0.0343\right)$ and IFN- $\gamma$ levels (CsHSP70: $t_{(2)}=$ 4.85, $P=0.04$; CsHSP90: $t_{(2)}=23.83, P=0.0018$ ) in T cells were increased relative to the PBS-treated group in response to rCsHSPs stimulation, although IL-4 level was slightly increased in case of rCsHSP90-treatment $\left(t_{(2)}=\right.$ 10.15, $P=0.0096$ ) (Fig. 6b).

\section{rCsHSP70 and rCsHSP90 stimulate IFN- $\gamma$ secretion from splenocytes of immunized mice}

We also measured cytokines secreted from splenocytes after re-stimulation in rCsHSPs-immunized mice (Fig. 7). The levels of IFN- $\gamma$ produced in the splenocytes in response to rCsHSP70 $\left(t_{(8)}=7.757, \quad P<0.0001\right)$ and rCsHSP90 $\left(t_{(8)}=3.398, P=0.0094\right)$ stimulation were significantly higher in cells isolated from immunized mice

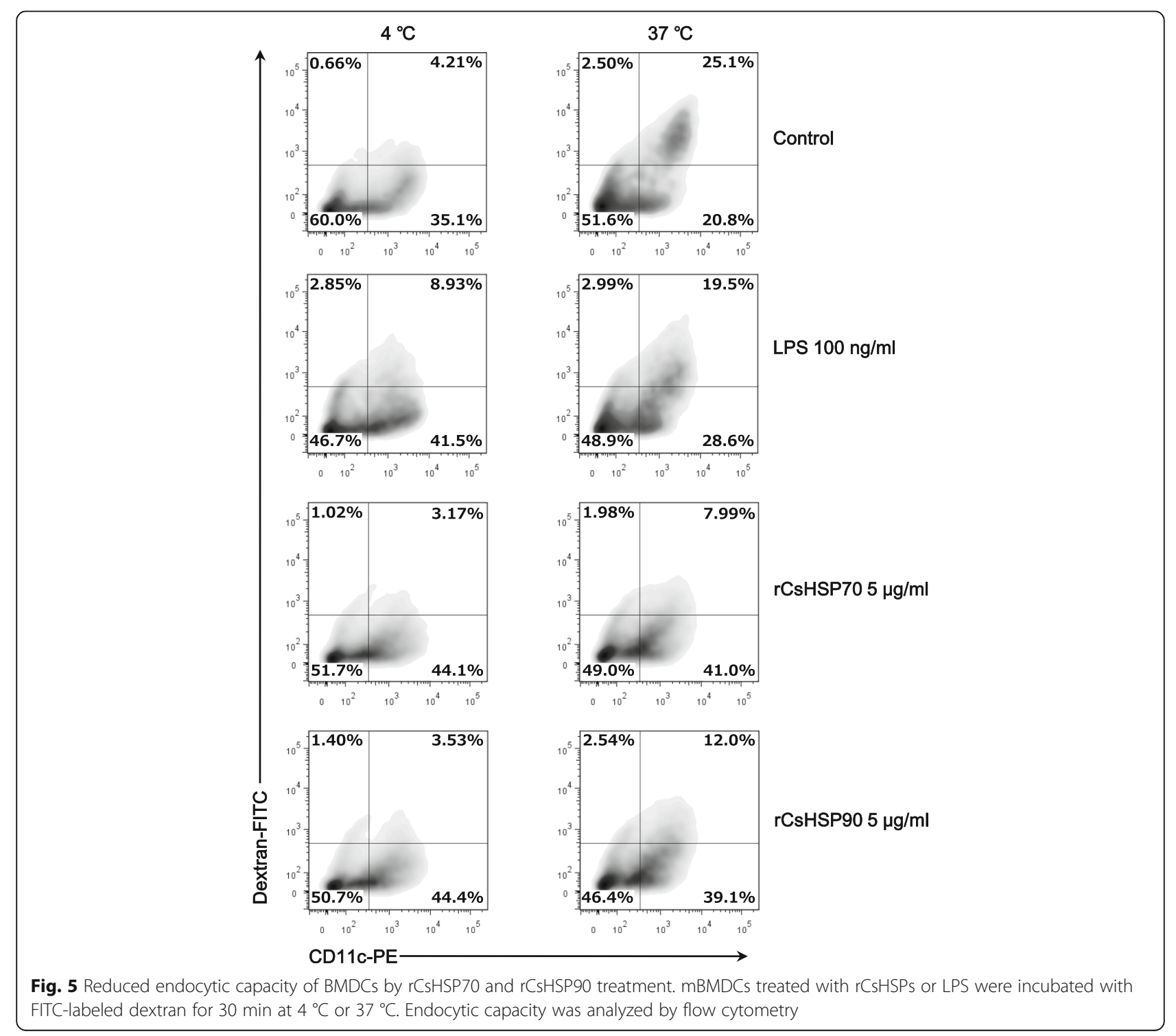




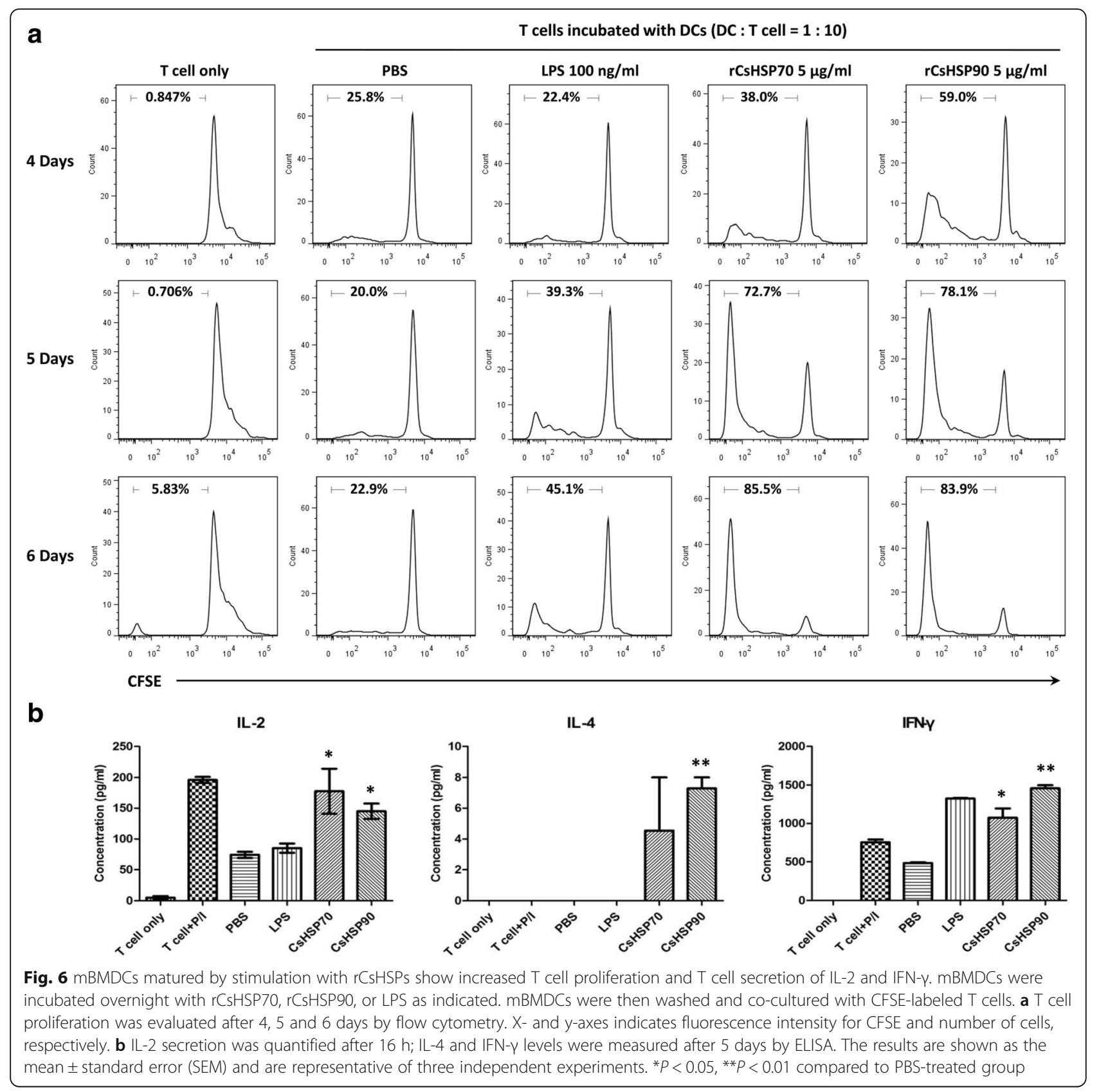

as compared to PBS-immunized control mice. But IL-2 and IL-4 were not changed. This result was consistent with the results of in vitro experiments.

\section{rCsHSPs/ProR peptide complexes increase cytokine secretion from $\mathrm{mBMDCs}$}

HSPs act as adjuvants in antigen presentation. To verify whether CsHSPs serve this function, we examined mBMDC activation by CsHSP70 or CsHSP90 bound to an antigenic peptide that cannot be presented as an antigen, by itself. ProR peptide of $C$. sinensis is a strong antigenic peptide that we previously detected in the sera of clonorchiasis patients (data not shown); we therefore tested whether the ProR peptide alone or in a complex with rCsHSP70 or rCsHSP90 could induce cellular immunity. When mBMDCs were stimulated with ProR peptide alone, surface molecule expression increased only at high concentration (Fig. 8) and cytokine secretion were unaltered (Fig. 9). However, CsHSP70/ProR peptide complexes increased TNF- $\alpha\left(t_{(2)}=4.501, P=0.046\right)$ and IL-6 $\left(t_{(2)}=9.817, P=0.0102\right)$ and $-1 \beta\left(t_{(2)}=40.91, P=0.0006\right)$ secretion from mBMDCs, and CsHSP90/ProR peptide complexes also stimulate IL-6 $\left(t_{(2)}=12.66, P=0.0062\right)$ and $-1 \beta\left(t_{(2)}=4.821, P=0.0404\right)$ secretion by mBMDCs (Fig. 9$)$, 


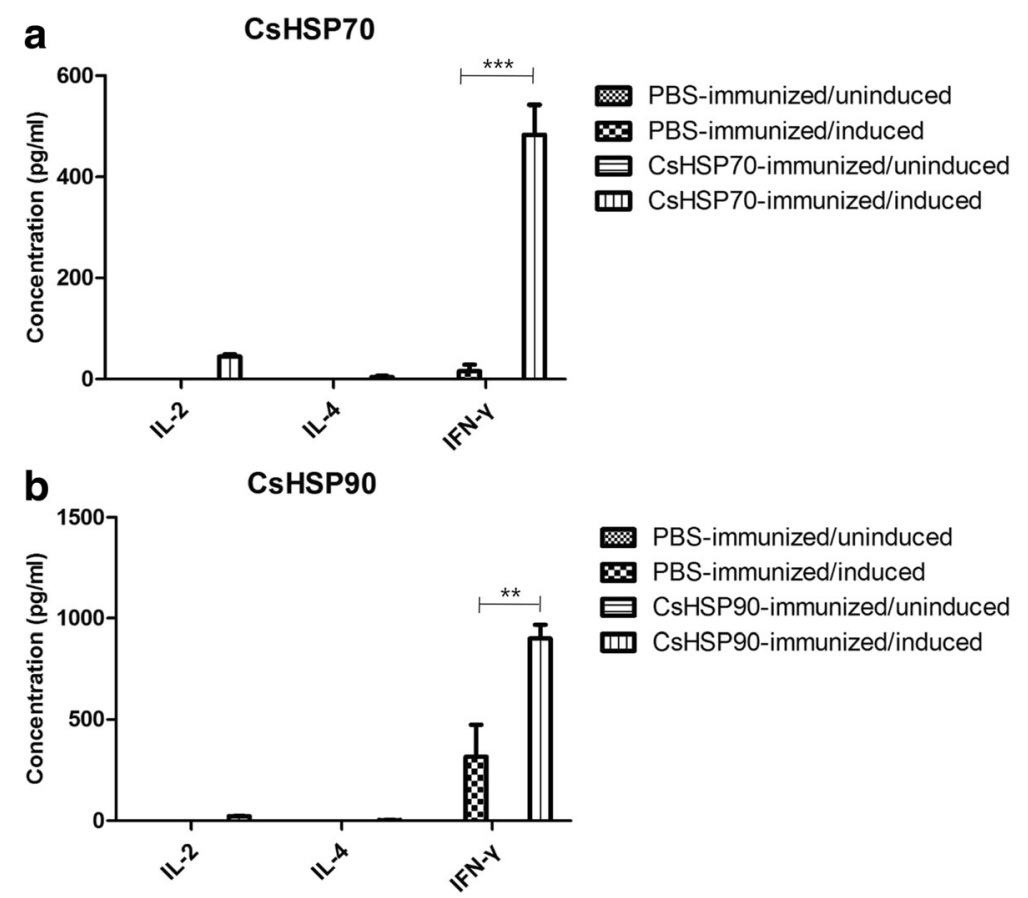

Fig. 7 Cytokines secreted from splenocytes of PBS or

rCSHSPs-immunized mice. Mice were immunized on day 0 with PBS or $100 \mu \mathrm{g}$ rCsHSPs per mouse emulsified in complete Freund's adjuvant followed by two booster injections with $100 \mu \mathrm{g}$ rCsHSPs per mouse emulsified in incomplete Freund's adjuvant on day 14th and 28th days. After the 7th day of the last immunization, the splenocytes were isolated from mice and were cultured either in the absence or in the presence of $5 \mu \mathrm{g} / \mathrm{ml}$ rCSHSP70 (a), rCSHSP90 (b) for $72 \mathrm{~h}$. The results are shown as the mean \pm standard error (SEM) and are representative of three independent experiments. ${ }^{* *} P<0.01,{ }^{* * *} P<0.001$

although surface marker expression was unaffected (data not shown).

\section{rCsHSPs stimulate CsProR peptide-specific antibody production}

We next examined whether CsHSP70 and CsHSP90 induce production of peptide-specific antibodies. To assess in vivo immune responses, ProR peptide was mixed with rCsHSPs or FA and used to immunize mice. $T$ cells isolated from the splenocytes of immunized mice were re-stimulated with $10 \mu \mathrm{g} / \mathrm{ml}$ ProR peptide, and anti-ProR antibody levels were measured in mouse serum. The $\mathrm{T}$ cell proliferation test revealed that the number of $\mathrm{T}$ cells did not increase in mice immunized with the peptide mixed with rCsHSPs or FA (Fig. 10a). However, the production of antibodies against ProR peptide was enhanced in mice immunized with CsHSP70/ProR peptide $\left(F_{(7,7)}=86.69, P<\right.$ $0.0001)$ or CsHSP90/ProR peptide $\left(F_{(7,7)}=18.51, P=\right.$ 0.001 ) complexes (Fig. 10b), with a stronger effect observed for rCsHSP70 than for rCsHSP90. Moreover, to determine the type of immune response, the isotype IgG1 and IgG2a was determined using ELISA. The results revealed that IgG1 levels were higher than IgG2a levels in both $\mathrm{rCsHSP70/ProR}\left(F_{(3,3)}=58.91, P=\right.$
$0.0073)$ and $\mathrm{rCsHSP90/ProR}\left(F_{(3,3)}=15.21, \quad P=0.05\right)$ immunized (Fig. 10c). This result indicates that rCsHSP/ProR complexes polarize the response towards the Th2 immune response in mice. Antibody isotyping results showed that rCsHSPs can induce Th2 immune response as well as Th1 response effectively.

\section{Discussion}

HSP acts as a molecular chaperone that enhance host immune responses [15, 16], and represent dominant antigens in many infectious diseases. Given their importance in host-pathogen immune interactions, these proteins are considered as vaccine candidates [35]. The present study showed that two recombinant HSPs derived from $C$. sinensis, rCsHSP70 and rCsHSP90, can stimulate murine DCs and T cells, as evidenced by the increase in co-stimulatory molecule expression and cytokine secretion in the presence of these proteins. We also found that $\mathrm{rCsHSPs}$ can induce production of antigenic peptide-specific antibodies.

Recombinant HSPs are often contaminated with bacteria-derived endotoxins, which are responsible for most in vitro cytokine effects of HSPs [16, 36]. However, the immunogenicity of HSPs with low endotoxin levels in DCs has also been reported $[18,19]$. Commonly used 


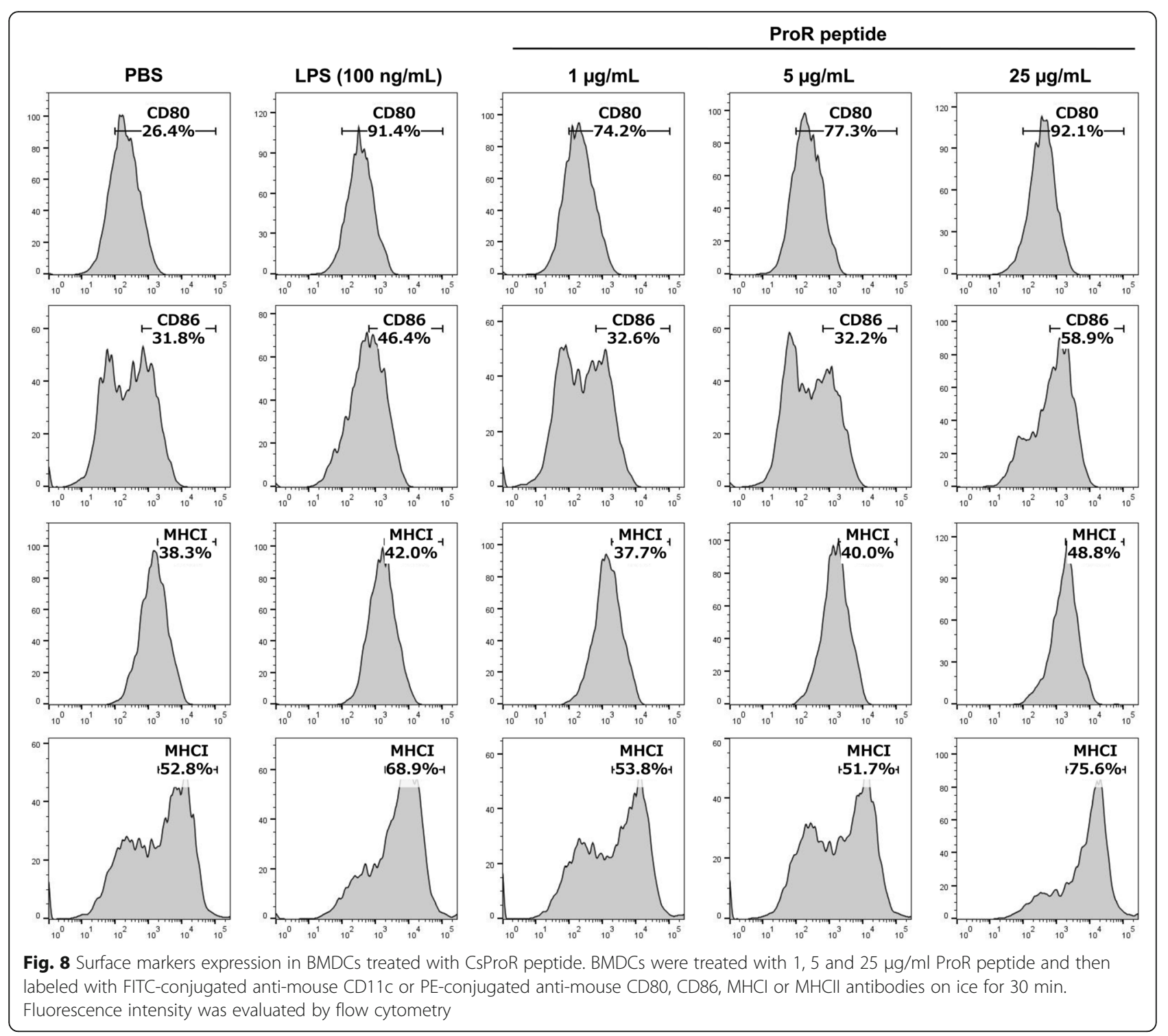

methods for eliminating endotoxin contamination in experiments using immune cells include endotoxin measurement using the LAL assay, and co-incubation of recombinant HSPs with the neutralizing agent polymyxin B [37]. In this study, we removed endotoxins from $\mathrm{rCsHSPs}$ with the LAL assay (endotoxin level $<1$ $\mathrm{EU} / \mathrm{ml}$ ); as an added precaution, the proteins were pre-incubated with polymyxin B before they were used in experiments.

Pathogen-derived HSPs induce an innate immune response that includes expression of surface markers on DCs and secretion of inflammatory cytokines [20, 38]. We examined the expression of the co-stimulatory molecules CD40, CD80, and CD86 as well as MHC I and MHC II as evidence of DC activation. Both rCsHSP70 and rCsHSP90 stimulated surface molecule expression and cytokine secretion (i.e., IL-1 $\beta$ and -6 and TNF- $\alpha$ ) in mBMDCs in a dose-dependent manner. High concentrations of rCsHSPs $(\geq 5 \mu \mathrm{g} / \mathrm{ml} \mathrm{CsHSP90}$ and $\geq 25 \mu \mathrm{g} / \mathrm{ml} \mathrm{CsHSP70)}$ also induced the secretion of IL-12 and -10 relative to PBS-treated control groups. In addition, mBMDCs acquired the capacity for production of intracellular IL-12 but not IL-10 in the presence of rCsHSPs. These results suggest that IL-10 is secreted early in the immune response. It is assumed that the capacity of DCs to capture and present antigens is diminished after initial activation [39]. We confirmed that both rCsHSP70 and rCsHSP90 stimulate cellular uptake and antigen presentation to mBMDCs. These results extend other reports that HSPs are potent activators of the innate immune system that can induce co-stimulatory molecule expression and pro-inflammatory cytokine secretion in mBMDCs $[19,20]$. 

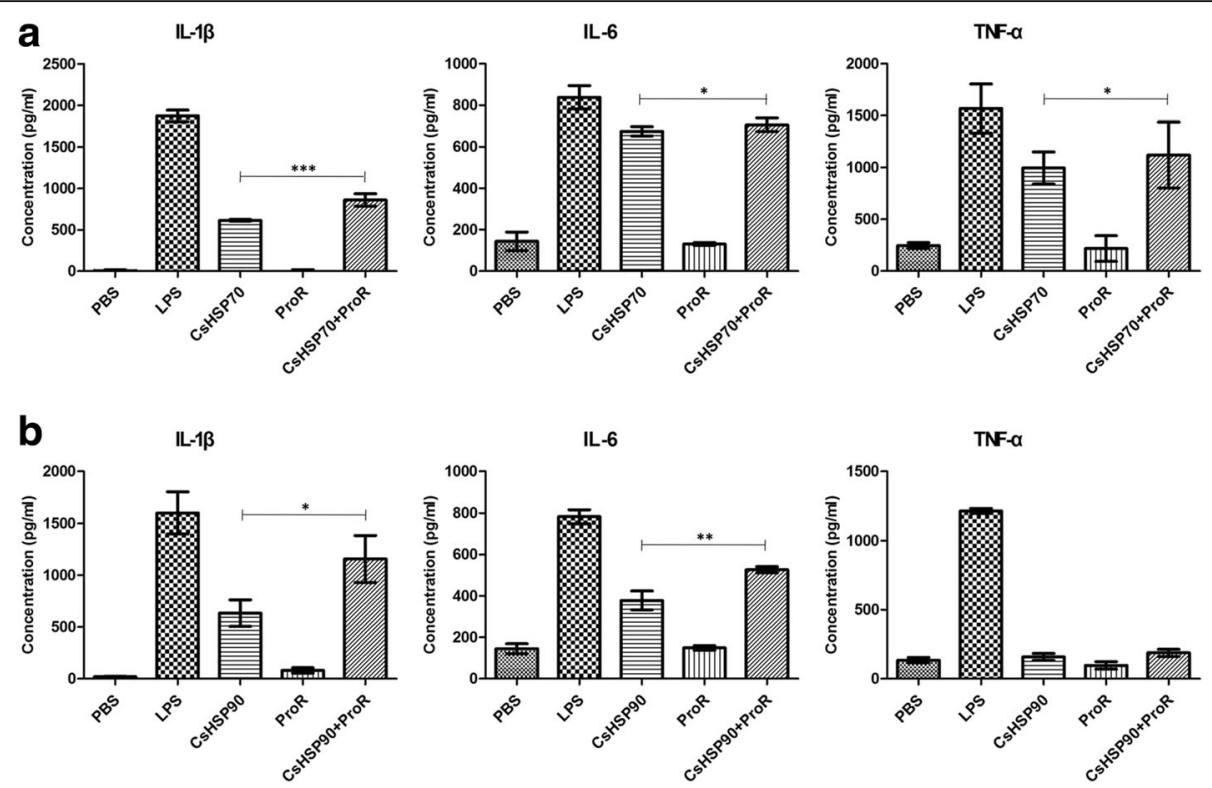

Fig. 9 Cytokines secretion by BMDCs induced by CsHSP/ProR peptide complexes. rCsHSP70 or rCsHSP90 (1 $\mu \mathrm{g} / \mathrm{ml})$ was mixed with ProR peptide at a 200:1 peptide:protein molar ratio in binding buffer at $37^{\circ} \mathrm{C}$ for $1 \mathrm{~h}$. After polymyxin B treatment for $1 \mathrm{~h}$, BMDCs were treated with rCsHSP70, rCsHSP90, CsProR peptide or CsHSP/ProR complexes. Levels of secreted cytokines were measured by ELISA. Panel a shows results by CsHSP70/ CsProR and panel b shows results by CsHSP90/CsProR. The results are shown as the mean \pm standard error (SEM). ${ }^{*} P<0.05,{ }^{* *} P<0.01,{ }^{* * *} P<0.001$
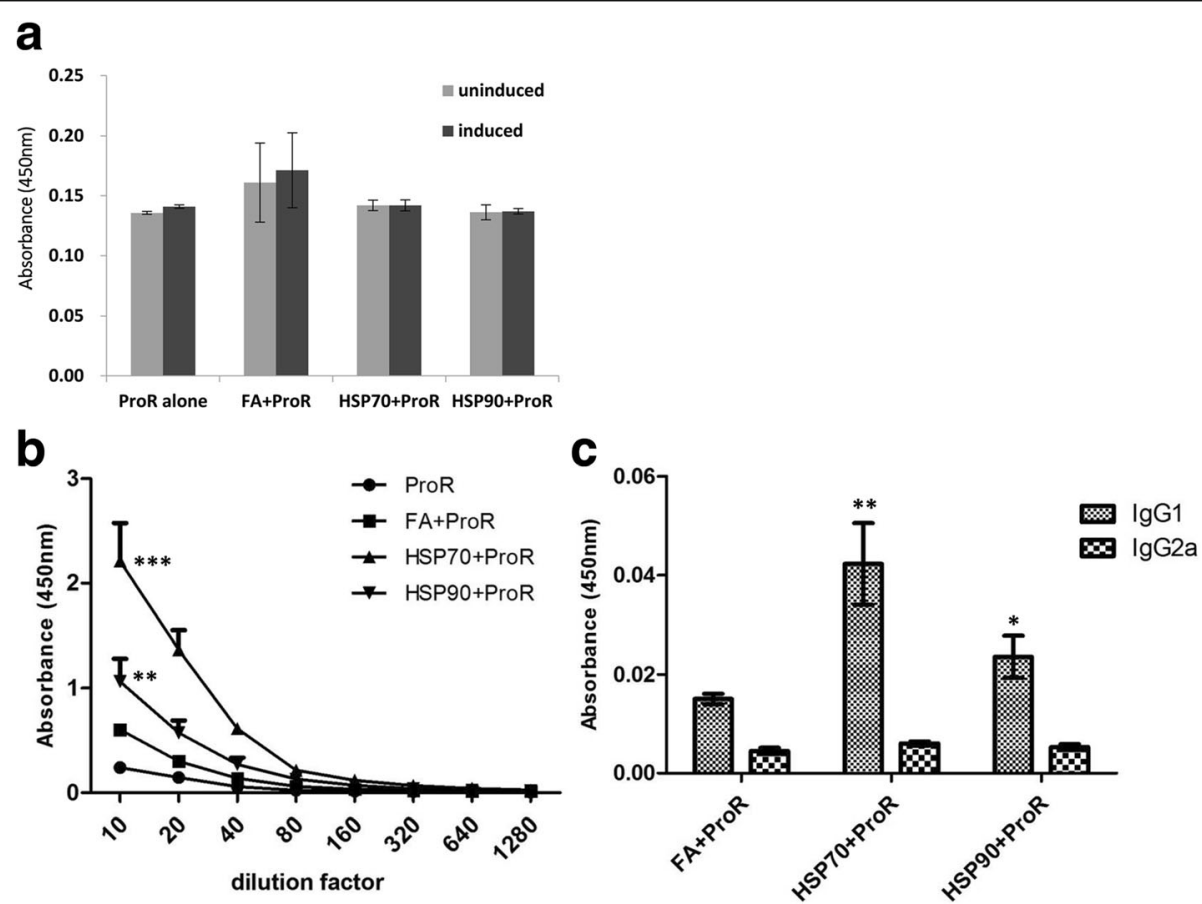

Fig. 10 rCsHSPs stimulate the production of anti-CsProR peptide antibodies via peptide interaction. Freund's adjuvant (FA) or rCsHSPs ( $30 \mu \mathrm{g} / \mathrm{ml}$ ) was incubated with CsProR peptide $(100 \mathrm{\mu g} / \mathrm{ml})$ for $1 \mathrm{~h}$ at room temperature. C57BL/6 mice were immunized with peptide immersed in FA or rCsHSP/peptide complexes. a T cells and sera were obtained from immunized mice on day 7 after final immunization. T cells were treated with PBS or $10 \mu \mathrm{g} / \mathrm{ml}$ CsProR peptide for $72 \mathrm{~h}$, and proliferation was then evaluated by WST-1 assay. Anti-CSProR antibodies (b) and their isotypes (c) detected in immunized mice serum by ELISA. The results are shown as the mean \pm standard error (SEM). ${ }^{*} P<0.05,{ }^{* *} P<0.01$, ${ }^{* *} P<0.001$ compared to PBS-immunized mice group 
To evaluate $\mathrm{T}$ cell activation by rCsHSPs, mBMDCs activated with rCsHSPs were co-cultured with allogenic $\mathrm{T}$ cells. The number of $\mathrm{T}$ cells activated with rCsHSPs increased over time, and secretion of IL- 2 and IFN- $\gamma$ but not IL-4 was also increased in T cells obtained from allogenic as compared to PBS-treated control cells. IFN- $\gamma$ was also secreted from splenocytes of rCsHSPs-immunized mice. These results demonstrate that $\mathrm{rCsHSP70}$ and rCsHSP90 mainly induce the Th1 immune response involving $\mathrm{CD}^{+} \mathrm{T}$ cells or macrophages, which could potentially disable parasites.

HSPs conjugated with peptide have been shown to induce $C D 8^{+}$CTL responses $[40,41]$. It has been reported that bacterial peptides elicit greater immune responses when bound to HSP [42]. In case of HSP from plants, HSP/antigen complexes have been shown to increase production of antibodies against a conjugated reporter antigen [43]. The Proline-rich (ProR) antigen of $C$. sinensis has been identified as a potential antigen [44]. In previous study, we identified that ProR peptide containing repeated sequences which derived from ProR antigen has strong antigenicity for clonorchiasis patients' sera (data not shown). To ascertain the activity of CsHSP70 and CsHSP90 as an adjuvant for antigenic peptide, we designed the complex of CsHSPs bound with ProR peptides. Here we found that ProR peptide alone did not induce activation of mBMDCs. We speculated that $\mathrm{rCsHSP} / \mathrm{ProR}$ peptide complexes would effectively induce an immune response, and generated CsHSP/ProR peptide complexes using ADP which increases peptide binding to HSP [18]. Incubation with CsHSP/ProR peptide complexes stimulated the secretion of TNF- $\alpha$, IL- 6 , and especially IL- $1 \beta$ by mBMDCs but had no effect on surface marker expression. We also immunized C57BL/6 mice with CsHSP/ProR peptide complexes and measured antibody production and $\mathrm{T}$ cell proliferation. The peptide alone or mixed with commercial adjuvant did not induce a peptide-specific antibody response in mice, consistent with another report [45]. However, antiProR peptide antibody production was increased upon immunization with rCsHSP/ProR. Although rCsHSP90 more strongly stimulated mBMDCs and $\mathrm{T}$ cells in vitro, the in vivo antibody response was greater against rCsHSP70-bound peptide. On the other hand, T cell proliferation was not induced by CsHSP/peptide complex immunization. Nonetheless, our findings suggest that CsHSPs have an adjuvant effect.

\section{Conclusions}

In conclusion, we confirmed that rCsHSP70 and rCsHSP90 are antigenic vaccine candidates since they activated the host immune response through cellular uptake and induction of antigen presentation. We also showed that CsHSPs can induce an in vitro immune response to ProR peptide derived from $C$. sinensis and have an adjuvant effect that includes the production of antibodies against ProR peptide. Our findings suggest that immunization with CsHSPpeptide conjugates can be an effective strategy for preventing C. sinensis infection or maturation.

\begin{abstract}
Abbreviations
APC: Antigen-presenting cell; APC: Allophycocyanin; CD: Cluster of differentiation; CFSE: Carboxyfluorescein succinimidyl ester; CTL: Cytotoxic T lymphocyte; DPBS: Dulbecco's phosphate buffered saline; ELISA: Enzyme-linked immunosorbent assay; FA: Freund's adjuvant; FBS: Fetal bovine serum; FITC: Fluorescein isothiocyanate; GM-CSF: Granulocyte-macrophage colony-stimulating factor; HSP: Heat shock protein; IFN: Interferon; IL: Interleukin; mBMDC: mouse bone marrow dendritic cell; $\mathrm{MHC}$ : Major histocompatibility complex; PE: Phycoerythrin; TNF: Tumor necrosis factor
\end{abstract}

\section{Acknowledgements}

This research was supported by an intramural research fund (2012-N54002-00) and a grant from the Pathogenic Proteome Management Program (NIH 4800-4847-311) from the National Research Institute of Health, Centers for Disease Control and Prevention, Ministry of Health, and Welfare, Republic of Korea.

\section{Funding}

Not applicable.

\section{Availability of data and materials}

The data supporting the conclusions of this article are included in the article.

\section{Authors' contributions}

EJC, YIJ and JWJ designed the study. EJC performed the experiments, analyzed the data and drafted the manuscript. MRL and YJK did the protein analysis. SEL, SHC, WJL and MYP performed the study design, and manuscript revision. All authors read and approved the final manuscript.

\section{Competing interests}

The authors declare that they have no competing interests.

\section{Consent for publication}

Not applicable.

\section{Ethics approval and consent to participate}

All animal experiments were approved by the Committee on the Ethics of Animal Experiments of the Korean Centers for Disease Control \& Prevention (permit number KCDC-171-14-2A). Experimental animals were maintained and handled in strict accordance with institutional guidelines of the Committee on the Ethics of Animal Experiments of the Korean Centers for Disease Control \& Prevention.

Received: 23 August 2016 Accepted: 9 February 2017

Published online: 01 March 2017

\section{References}

1. Chai JY, Darwin Murrell K, Lymbery AJ. Fish-borne parasitic zoonoses: status and issues. Int J Parasitol. 2005;35(11-12):1233-54.

2. Lim JH. Liver flukes: the malady neglected. Korean J Radiol. 2011;12(3):269-79

3. Lun ZR, Gasser RB, Lai DH, Li AX, Zhu XQ, Yu XB, Fang YY. Clonorchiasis: a key foodborne zoonosis in China. Lancet Inf Dis. 2005;5(1):31-41.

4. Shin EH, Guk SM, Kim HJ, Lee SH, Chai JY. Trends in parasitic diseases in the Republic of Korea. Trends Parasitol. 2008;24(3):143-50.

5. Zhang GW, Lin JH, Qian JP, Zhou J. Identification of risk and prognostic factors for patients with clonorchiasis-associated intrahepatic cholangiocarcinoma. Ann Surg Oncol. 2014;21(11):3628-37.

6. Shin HR, Oh JK, Masuyer E, Curado MP, Bouvard V, Fang YY, et al. Epidemiology of cholangiocarcinoma: an update focusing on risk factors. Cancer Sci. 2010;101(3):579-85.

7. Furst T, Keiser J, Utzinger J. Global burden of human food-borne trematodiasis: a systematic review and meta-analysis. Lancet Inf Dis. 2012;12(3):210-21. 
8. Cho SH, Lee KY, Lee BC, Cho PY, Cheun HI, Hong ST, et al. Prevalence of clonorchiasis in southern endemic areas of Korea in 2006. Korean J Parasitol. 2008;46(3):133-7.

9. Watanapa P, Watanapa WB. Liver fluke-associated cholangiocarcinoma. Br J Surg. 2002;89(8):962-70.

10. Blechacz B, Gores GJ. Cholangiocarcinoma: advances in pathogenesis, diagnosis, and treatment. Hepatology. 2008;48(1):308-21.

11. Qian MB, Chen YD, Liang S, Yang GJ, Zhou XN. The global epidemiology of clonorchiasis and its relation with cholangiocarcinoma. Infect Dis Poverty. 2012;1(1):4.

12. Kim HG, Han J, Kim MH, Cho KH, Shin IH, Kim GH, et al. Prevalence of clonorchiasis in patients with gastrointestinal disease: a Korean nationwide multicenter survey. World J Gastroenterol. 2009;15(1):86-94.

13. Wang X, Chen W, Li X, Zhou C, Deng C, Lv X, et al. Identification and molecular characterization of a novel signaling molecule 14-3-3 epsilon in Clonorchis sinensis excretory/secretory products. Parasitol Res. 2012:110(4):1411-20.

14. Zhou Z, Xia H, Hu X, Huang Y, Li Y, Li L, et al. Oral administration of a Bacillus subtilis spore-based vaccine expressing Clonorchis sinensis tegumental protein $22.3 \mathrm{kDa}$ confers protection against Clonorchis sinensis. Vaccine. 2008;26(15):1817-25.

15. McNulty S, Colaco CA, Blandford LE, Bailey CR, Baschieri S, Todryk S. Heat-shock proteins as dendritic cell-targeting vaccines - getting warmer. Immunology. 2013;139(4):407-15.

16. Tsan M-F, Gao B. Heat shock proteins and immune system. J Leukoc Biol. 2009;85(6):905-10.

17. Habich C, Kempe K, van der Zee R, Rumenapf R, Akiyama H, Kolb H, Burkart $\checkmark$. Heat shock protein 60: specific binding of lipopolysaccharide. J Immunol. 2005;174(3):1298-305.

18. Moroi Y, Mayhew M, Trcka J, Hoe MH, Takechi Y, Hartl FU, et al. Induction of cellular immunity by immunization with novel hybrid peptides complexed to heat shock protein 70. Proc Natl Acad Sci USA. 2000;97(7):3485-90.

19. Ashtekar AR, Zhang P, Katz J, Deivanayagam CC, Rallabhandi P, Vogel SN, Michalek SM. TLR4-mediated activation of dendritic cells by the heat shock protein DnaK from Francisella tularensis. J Leukoc Biol. 2008;84(6):1434-46.

20. Fang L, Sun L, Yang J, Gu Y, Zhan B, Huang J, Zhu X. Heat shock protein 70 from Trichinella spiralis induces protective immunity in BALB/C mice by activating dendritic cells. Vaccine. 2014;32(35):4412-9.

21. Breloer M, Dorner B, More SH, Roderian T, Fleischer B, von Bonin A. Heat shock proteins as "danger signals": eukaryotic Hsp60 enhances and accelerates antigen-specific IFN-gamma production in T cells. Eur J Immunol. 2001;31(7):2051-9.

22. More SH, Breloer M, von Bonin A. Eukaryotic heat shock proteins as molecular links in innate and adaptive immune responses: Hsp60-mediated activation of cytotoxic T cells. Int Immunol. 2001;13(9):1121-7.

23. Binder RJ. Heat-shock protein-based vaccines for cancer and infectious disease. Expert Rev Vaccines. 2008;7(3):383-93.

24. SenGupta D, Norris PJ, Suscovich TJ, Hassan-Zahraee M, Moffett HF, Trocha A, et al. Heat shock protein-mediated cross-presentation of exogenous HIV antigen on HLA class I and class II. J Immunol. 2004;173(3):1987-93.

25. Tobian AA, Canaday DH, Harding CV. Bacterial heat shock proteins enhance class II MHC antigen processing and presentation of chaperoned peptides to CD4+ T cells. J Immunol. 2004;173(8):5130-7.

26. Pockley AG. Heat shock proteins as regulators of the immune response. Lancet. 2003;362(9382):469-76.

27. Navaratnam M, Deshpande MS, Hariharan MJ, Zatechka Jr DS, Srikumaran S. Heat shock protein-peptide complexes elicit cytotoxic T-lymphocyte and antibody responses specific for bovine herpesvirus 1. Vaccine. 2001:19(11-12):1425-34.

28. Hartmann W, Singh N, Rathaur S, Brenz Y, Liebau E, Fleischer B, Breloer M. Immunization with Brugia malayi Hsp70 protects mice against Litomosoides sigmodontis challenge infection. Parasite Immunol. 2014;36(4):141-9.

29. He S, Yang L, Lv Z, Hu W, Cao J, Wei J, et al. Molecular and functional characterization of a mortalin-like protein from Schistosoma japonicum (SjMLP/hsp70) as a member of the HSP70 family. Parasitol Res. 2010;107(4):955-66.

30. Dakshinamoorthy G, Samykutty AK, Munirathinam G, Reddy MV Kalyanasundaram R. Multivalent fusion protein vaccine for lymphatic filariasis. Vaccine. 2013;31(12):1616-22.
31. Wang S, Zhu X, Yang Y, Yang J, Gu Y, Wei J, et al. Molecular cloning and characterization of heat shock protein 70 from Trichinella spiralis. Acta Trop. 2009;110(1):46-51.

32. Flohe SB, Bruggemann J, Lendemans S, Nikulina M, Meierhoff G, Flohe S, Kolb H. Human heat shock protein 60 induces maturation of dendritic cells versus a Th1-promoting phenotype. J Immunol. 2003;170(5):2340-8.

33. Kim SK, Im J, Yun CH, Son JY, Son CG, Park DK, Han SH. Armillariella mellea induces maturation of human dendritic cells without induction of cytokine expression. J Ethnopharmacol. 2008:119(1):153-9.

34. Garrett WS, Chen LM, Kroschewski R, Ebersold M, Turley S, Trombetta S, et al. Developmental control of endocytosis in dendritic cells by $\mathrm{Cdc} 42$. Cell. 2000;102(3):325-34

35. Zugel U, Kaufmann SH. Immune response against heat shock proteins in infectious diseases. Immunobiology. 1999;201(1):22-35.

36. Motta A, Schmitz C, Rodrigues L, Ribeiro F, Teixeira C, Detanico T, et al. Mycobacterium tuberculosis heat-shock protein 70 impairs maturation of dendritic cells from bone marrow precursors, induces interleukin-10 production and inhibits T-cell proliferation in vitro. Immunology. 2007:121(4):462-72.

37. Wallin RP, Lundqvist A, More SH, von Bonin A, Kiessling R, Ljunggren HG. Heat-shock proteins as activators of the innate immune system. Trends Immunol. 2002;23(3):130-5

38. Tsan MF, Gao B. Heat shock protein and innate immunity. Cell Mol Immunol. 2004;1(4):274-9.

39. Platt CD, Ma JK, Chalouni C, Ebersold M, Bou-Reslan H, Carano RA, et al. Mature dendritic cells use endocytic receptors to capture and present antigens. Proc Natl Acad Sci USA. 2010;107(9):4287-92.

40. Flechtner JB, Cohane KP, Mehta S, Slusarewicz P, Leonard AK, Barber BH, et al. High-affinity interactions between peptides and heat shock protein 70 augment CD8+ T lymphocyte immune responses. J Immunol. 2006:177(2):1017-27.

41. Blachere NE, Li Z, Chandawarkar RY, Suto R, Jaikaria NS, Basu S, et al. Heat shock protein-peptide complexes, reconstituted in vitro, elicit peptidespecific cytotoxic T lymphocyte response and tumor immunity. J Exp Med. 1997:186(8):1315-22

42. MacAry PA, Javid B, Floto RA, Smith KG, Oehlmann W, Singh M, Lehner PJ. HSP70 peptide binding mutants separate antigen delivery from dendritic cell stimulation. Immunity. 2004;20(1):95-106.

43. Corigliano MG, Fenoy I, Sander V, Maglioco A, Goldman A, Clemente M. Plant heat shock protein 90 as carrier-adjuvant for immunization against a reporter antigen. Vaccine. 2013;31(49):5872-8.

44. Kim TY, Kang SY, Ahn IY, Cho SY, Hong SJ. Molecular cloning and characterization of an antigenic protein with a repeating region from Clonorchis sinensis. Korean J Parasitol. 2001;39(1):57-66.

45. Qian F, Guo A, Li M, Liu W, Pan Z, Jiang L, et al. Salmonella flagellin is a potent carrier-adjuvant for peptide conjugate to induce peptide-specific antibody response in mice. Vaccine. 2015;33(17):2038-44.

\section{Submit your next manuscript to BioMed Central and we will help you at every step:}

- We accept pre-submission inquiries

- Our selector tool helps you to find the most relevant journal

- We provide round the clock customer support

- Convenient online submission

- Thorough peer review

- Inclusion in PubMed and all major indexing services

- Maximum visibility for your research

Submit your manuscript at www.biomedcentral.com/submit 